\title{
Improved LDA Classifier based on Spiked Models
}

\author{
Houssem Sifaou, Abla Kammoun, Mohamed-Slim Alouini \\ Computer, Electrical, and Mathematical Sciences \& Engineering Division, \\ King Abdullah University of Science and Technology, Thuwal, Saudi Arabia \\ Email: \{houssem.sifaou, abla.kammoun, slim.alouini\}@kaust.edu.sa
}

\begin{abstract}
Linear discriminant analysis (LDA) is a popular technique for classification that is built on the assumption that data follow a Gaussian mixture model with a common covariance matrix. A major challenge towards the use of LDA in practice is that the classifier depends on the mean parameters and the inverse of covariance matrix of the Gaussian mixture model that need to be estimated from training data. Several estimators for the inverse of the covariance matrix can be used. The most common ones are estimators based on the regularization approach, giving the name regularized LDA (R-LDA) to the corresponding classifier. The main advantage of such estimators is their resilience to the sampling noise, making them suitable to high dimensional settings. In this paper, we propose a new estimator that is shown to yield better classification performance than the classical R-LDA. The main principle of our proposed method is the design of an optimized inverse covariance matrix estimator based on the assumption that the true covariance matrix is a low-rank perturbation of a scaled identity matrix. We show that not only the proposed classifier is easier to implement but also, as evidenced by numerical experiments, it outperforms the LDA and R-LDA classifiers for both real and synthetic data.
\end{abstract}

Index Terms-Linear discriminant analysis, spiked models, high dimensional data, random matrix theory.

\section{INTRODUCTION}

Linear Discriminant Analysis (LDA) based classifier has been widely used since it was originally proposed by R. A. Fisher [1]. It has been applied in several fields such as taxonomic problems [1], detection [2], face and speech recognition [3], [4], and cancer genomics [5]. Its popularity owes to its yielding the least classification error rate under the assumption that data follow a Gaussian mixture model with a common covariance matrix [6].

One major difficulty when it comes to the practical use of LDA lies in that it involves unknown statistics, namely the inverse of the population covariance matrix and the mean vectors associated with each class. To solve this issue, a widely adopted approach consists in replacing the population covariance matrix and the mean of the classes by their empirical estimates, obtained from available training data. While this approach is expected to yield little loss in classification accuracy when the number of training samples is very high compared to their dimensions, it becomes highly inaccurate in high dimensional settings where the number of observations is of the same order as the sample size. This becomes all the more problematic that current practical scenarios require to cope with high dimensional data sets. One popular solution to solve this issue are classifiers that use the regularized sample covariance matrix as a plug-in estimator of the population covariance matrix. The resulting classifier is known as the Regularized LDA (R-LDA) in reference to the regularization parameter in use. However, a key question is how to choose the regularization parameter. A series of recent works propose to set the regularization parameter to the value that minimizes the asymptotic classification error, which can be derived using recent results from random matrix theory [7]-[9]. Such an approach presents two major drawbacks. First, the estimation of the optimal regularization parameter is computationally expensive. Second, it does not take advantage from available prior information on the structure of the covariance matrix.
In this paper, we propose an improved LDA classifier that is suited to scenarios in which the population covariance matrix is isotropic except for finite number of symmetry breaking directions [10], [11]. Such a model, which we refer to as a spiked-model covariance matrix, is found to be relevant to many real applications including detection, EEG signals and financial econometrics. Based on this model, we propose to consider a class of estimators that take the form of a finite rank perturbation of a scaled identity matrix. The directions of the perturbation are given by the principal eigenvectors of the sample covariance matrix, while its eigenvalues are design parameters to be optimized. Using results from random matrix theory, we derive the asymptotic classification error rate of the LDA classifier that uses this estimator in place of the population covariance matrix. This result is then used to optimize the design parameters in order to minimize the misclassification error rate. The solution of such a problem is easy to compute and leads to a novel improved classifier which we refer to as "Imp-LDA" classifier. The performance of the proposed classifier is assessed through a set of experiments carried out for both synthetic and real data. Interestingly, we demonstrate that the improved classifier, not only avoids the grid search often required in the determination of the regularization parameter of R-LDA [6], [7], [12], but also presents better classification performance, making it an attractive classification method well-suited to high dimensional settings.

The rest of the paper is organized a follows. The next section revisits the LDA and R-LDA classifiers. In section III, our improved LDA classifier is presented. Numerical simulations are provided in section IV prior to concluding in section V.

Throughout this work, boldface lower case is used for denoting column vectors, $\mathbf{x}$, and upper case for matrices, $\mathbf{X} . \mathbf{X}^{T}$ denotes the transpose. Moreover, $\mathbf{I}_{p}$ denotes the $p \times p$ identity matrix and $\|\cdot\|$ is used to denote the $\ell_{2}$ norm.

\section{LINEAR DISCRIMINANT ANALYSIS}

Consider a set of $n$ vector observations $\mathbf{x}_{1}, \cdots, \mathbf{x}_{n}$ in $\mathbb{R}^{p \times 1}$ belonging to two classes $\mathcal{C}_{0}$ and $\mathcal{C}_{1}$. For $i \in\{0,1\}$, we denote by $n_{i}$ the number of observations in $\mathcal{C}_{i}$ and by $\mathcal{T}_{i}$ the set of their indexes. Moreover, all observations are assumed to be independent and drawn from a Gaussian mixture model in which both classes have different means but a common covariance matrix. In particular, for $i \in\{0,1\}$,

$$
\mathbf{x}_{i} \in \mathcal{C}_{i} \Leftrightarrow \mathbf{x}_{i} \sim \mathcal{N}\left(\boldsymbol{\mu}_{i}, \boldsymbol{\Sigma}\right)
$$

where $\boldsymbol{\mu}_{i}$ is the mean vector associated with class $i$ and $\boldsymbol{\Sigma}$ is the covariance matrix common to both classes. The class association of each observation $\mathbf{x}_{\ell}$ is known. These observations constitute thus the training data set and are used to build a classifier aimed to be used on unseen data observations to predict their class label. Of interest in this work is the classical LDA classifier, the discriminant score of which is given by [6], [7], [12]:

$$
W^{\mathrm{LDA}}(\mathbf{x})=\left(\mathbf{x}-\frac{\overline{\mathbf{x}}_{0}+\overline{\mathbf{x}}_{1}}{2}\right)^{T} \hat{\mathbf{\Sigma}}^{-1}\left(\overline{\mathbf{x}}_{0}-\overline{\mathbf{x}}_{1}\right)-\log \frac{\pi_{1}}{\pi_{0}},
$$


with $\overline{\mathbf{x}}_{i}=\frac{1}{n_{i}} \sum_{\ell \in \mathcal{T}_{i}} \mathbf{x}_{\ell}$ is the empirical mean associated with class $i$ and $\hat{\boldsymbol{\Sigma}}$ is the pooled covariance matrix defined as

$$
\hat{\boldsymbol{\Sigma}}=\frac{\left(n_{0}-1\right) \hat{\boldsymbol{\Sigma}}_{0}+\left(n_{1}-1\right) \hat{\boldsymbol{\Sigma}}_{1}}{n-2},
$$

where

$$
\hat{\mathbf{\Sigma}}_{i}=\frac{1}{n_{i}-1} \sum_{\ell \in \mathcal{T}_{i}}\left(\mathbf{x}_{\ell}-\overline{\mathbf{x}}_{i}\right)\left(\mathbf{x}_{\ell}-\overline{\mathbf{x}}_{i}\right)^{T}, \quad i=0,1 .
$$

while $\pi_{i}$ is the prior probability corresponding to class $\mathcal{C}_{i}$. The observation $\mathbf{x}$ is thus assigned to $\mathcal{C}_{0}$ whenever $W^{\mathrm{LDA}}(\mathbf{x})>0$ and to class $\mathcal{C}_{1}$ otherwise. In the case where $p>n$, and to overcome singularity issues of $\hat{\boldsymbol{\Sigma}}$, ridge estimators of the inverse covariance matrix are used [6], [7] which yields the R-LDA classifier:

$$
W^{\mathrm{R}-\mathrm{LDA}}(\mathbf{x})=\left(\mathbf{x}-\frac{\overline{\mathbf{x}}_{0}+\overline{\mathbf{x}}_{1}}{2}\right)^{T} \mathbf{H}\left(\overline{\mathbf{x}}_{0}-\overline{\mathbf{x}}_{1}\right)-\log \frac{\pi_{1}}{\pi_{0}},
$$

where

$$
\mathbf{H}=\left(\mathbf{I}_{p}+\gamma \hat{\boldsymbol{\Sigma}}\right)^{-1}, \quad \gamma>0 .
$$

Conditioning on the training observations, the discriminant score $W^{\mathrm{LDA}}(\mathbf{x})$ is Gaussian in $\mathbf{x}$. In light of this observation, the conditional misclassification error rate associated with class $i$ can be expressed as:

$$
\epsilon_{i}^{\mathrm{LDA}}=\boldsymbol{\Phi}\left(\frac{(-1)^{i+1} G\left(\boldsymbol{\mu}_{i}, \overline{\mathbf{x}}_{0}, \overline{\mathbf{x}}_{1}, \hat{\boldsymbol{\Sigma}}\right)+(-1)^{i} \log \frac{\pi_{1}}{\pi_{0}}}{\sqrt{D\left(\boldsymbol{\mu}_{i}, \overline{\mathbf{x}}_{0}, \overline{\mathbf{x}}_{1}, \hat{\boldsymbol{\Sigma}}, \boldsymbol{\Sigma}\right)}}\right)
$$

where $\boldsymbol{\Phi}($.$) is the cumulative distribution function of a standard$ normal random variable and

$$
\begin{aligned}
& G\left(\boldsymbol{\mu}_{i}, \overline{\mathbf{x}}_{0}, \overline{\mathbf{x}}_{1}, \hat{\boldsymbol{\Sigma}}\right)=\left(\boldsymbol{\mu}_{i}-\frac{\overline{\mathbf{x}}_{0}+\overline{\mathbf{x}}_{1}}{2}\right) \hat{\boldsymbol{\Sigma}}^{-1}\left(\overline{\mathbf{x}}_{0}-\overline{\mathbf{x}}_{1}\right), \\
& D\left(\boldsymbol{\mu}_{i}, \overline{\mathbf{x}}_{0}, \overline{\mathbf{x}}_{1}, \hat{\boldsymbol{\Sigma}}, \boldsymbol{\Sigma}\right)=\left(\overline{\mathbf{x}}_{0}-\overline{\mathbf{x}}_{1}\right)^{T} \hat{\boldsymbol{\Sigma}}^{-1} \boldsymbol{\Sigma} \hat{\boldsymbol{\Sigma}}^{-1}\left(\overline{\mathbf{x}}_{0}-\overline{\mathbf{x}}_{1}\right) .
\end{aligned}
$$

The total classification error rate writes as:

$$
\epsilon^{\mathrm{LDA}}=\pi_{0} \epsilon_{0}^{\mathrm{LDA}}+\pi_{1} \epsilon_{1}^{\mathrm{LDA}} .
$$

Along the same arguments, the classification error rate associated with R-LDA takes the same expression with $\hat{\boldsymbol{\Sigma}}^{-1}$ being replaced by $\mathbf{H}$ in (6). This expression is however not instructive on how the theoretical mean and covariances of each class impact the classification performances. One approach to get around this issue is to use asymptotic results from random matrix theory which lead to approximate the quantities $G\left(\boldsymbol{\mu}_{i}, \overline{\mathbf{x}}_{0}, \overline{\mathbf{x}}_{1}, \hat{\boldsymbol{\Sigma}}\right)$ and $D\left(\boldsymbol{\mu}_{i}, \overline{\mathbf{x}}_{0}, \overline{\mathbf{x}}_{1}, \hat{\boldsymbol{\Sigma}}, \boldsymbol{\Sigma}\right)$ by deterministic equivalents that solely involve $\left\{\boldsymbol{\mu}_{i}\right\}_{i=1}^{2}$ and $\boldsymbol{\Sigma}$ [7], [13], [14]. A key question, however, is how to set the regularization parameter $\gamma$. As the misclassification error rate is one of the most important key performance metric in classification, it is natural to look for the regularization parameter that minimizes the total asymptotic classification error rate. One major difficulty toward achieving this goal lies in that the expression of the total misclassification error rate depends on the unknown covariance matrix $\boldsymbol{\Sigma}$ and $\left\{\boldsymbol{\mu}_{i}\right\}_{i=0}^{1}$. The works in [7], [9] propose consistent estimates of the misclassification error rates, which were then optimized through a grid search. This approach has been shown through simulations to outperform the classical cross-validation technique used often for the setting of the regularization parameter. It however presents two major drawbacks. First, the optimization of the regularization parameter involves a gridsearch procedure which can lead to prohibitively high computational costs when high dimension settings are considered. Second, it relies on the sample covariance matrix which might lead the classifier to undergo high estimation noises. In this paper, we propose an improved LDA classifier that overcomes both drawbacks. To this end, we assume that $\boldsymbol{\Sigma}$ takes a particular form [10], [11]:

$$
\boldsymbol{\Sigma}=\sigma^{2} \mathbf{I}_{p}+\sigma^{2} \sum_{j=1}^{r} \lambda_{j} \mathbf{u}_{j} \mathbf{u}_{j}^{T}
$$

where $\sigma^{2}>0, \lambda_{1} \geq \cdots, \geq \lambda_{r}>0$ and $\mathbf{u}_{1}, \cdots, \mathbf{u}_{r}$ are orthonormal. The above model is encountered in many real applications, among which detection, EEG signals and financial econometrics are the best representatives.

\section{IMPROVED LDA}

In this section, we propose our improved LDA classifier, which unlike the traditional R-LDA, leverages the particular finite rank perturbation property of the true covariance matrix. For the sake of simplicity, we assume that $\sigma^{2}$ and $r$ are perfectly known. In practice, one can resort to the existing efficient algorithms available in the literature for the estimation of these parameters. We refer the reader to the following works and the references therein [15]-[18]. Noting that:

$$
\boldsymbol{\Sigma}^{-1}=\frac{1}{\sigma^{2}}\left[\mathbf{I}_{p}-\sum_{j=1}^{r} \frac{\lambda_{j}}{1+\lambda_{j}} \mathbf{u}_{j} \mathbf{u}_{j}^{T}\right]
$$

it is sensible to seek for estimators of $\boldsymbol{\Sigma}^{-1}$ that takes the form:

$$
\hat{\mathbf{C}}^{-1}=\frac{1}{\sigma^{2}}\left[\mathbf{I}_{p}+\sum_{j=1}^{r} w_{j} \mathbf{v}_{j} \mathbf{v}_{j}^{T}\right]
$$

where $\mathbf{v}_{1}, \cdots, \mathbf{v}_{r}$ are the $r$ principal eigenvectors associated with the largest eigenvalues of the pooled covariance matrix $\hat{\boldsymbol{\Sigma}}$, and $\left\{w_{j}\right\}_{j=1}^{r}$ are some design parameters in $(-1,0)$ to be optimized. Plugging (8) in place of $\hat{\boldsymbol{\Sigma}}$ into (1), yields the following discriminant score:

$$
W^{\mathrm{Imp}-\mathrm{LDA}}=\left(\mathbf{x}-\frac{\overline{\mathbf{x}}_{0}+\overline{\mathbf{x}}_{1}}{2}\right)^{T} \hat{\mathbf{C}}^{-1}\left(\overline{\mathbf{x}}_{0}-\overline{\mathbf{x}}_{1}\right)-\log \frac{\pi_{1}}{\pi_{0}},
$$

It is worth mentioning that the estimator $\hat{\mathbf{C}}^{-1}$ can be seen as an instance of the wider class of estimators taking the form $\sum_{j=1}^{p} \eta_{j} \mathbf{v}_{j} \mathbf{v}_{j}^{T}$, where $\mathbf{v}_{j}$ being the eigenvector of the sample covariance matrix associated with its $j$-th largest eigenvalue and scalars $\eta_{j}$ are referred to as shrinkage functions that need to be designed [19]-[23]. Indeed, $\hat{\mathbf{C}}^{-1}$ is obtained by setting $\eta_{r+1}=\ldots=\eta_{p}=\frac{1}{\sigma^{2}}$ and $w_{j}=\sigma^{2} \eta_{j}-1$. The main challenge is how to select the appropriate values of the design parameters $w_{j}$. Given the application into consideration, it is sensible to select the $w_{j}$ so that they minimize the total classification error rate:

$$
\mathbf{w}^{\star}=\underset{\mathbf{w}}{\operatorname{argmin}} \mathbb{E}\left[\epsilon^{\mathrm{Imp}-\mathrm{LDA}}\right]
$$

where $\mathbf{w}=\left[w_{1}, \cdots, w_{r}\right]^{T}$ and $\epsilon^{\operatorname{Imp}-\mathrm{LDA}}$ is obtained by replacing $\hat{\boldsymbol{\Sigma}}^{-1}$ by $\hat{\mathbf{C}}^{-1}$ in (6) and the expectation is taken with respect to the training data. Finding the optimal $\mathbf{w}$ that exactly solves (9) could not be in general obtained. To get around this problem, we invoke results from random matrix theory that approximate the total misclassification error rate under the following asymptotic regime defined in the following assumption.

Assumption 1. $\quad n, p \longrightarrow \infty$, with fixed ratio $c=p / n$.

- $r$ is fixed and $\lambda_{1}>\cdots>\lambda_{r}>\sqrt{c}$, independently of $p$ and $n$.

Theorem 1. Under the settings of Assumption 1, we have

$$
G\left(\boldsymbol{\mu}_{i}, \overline{\mathbf{x}}_{0}, \overline{\mathbf{x}}_{1}, \hat{\mathbf{C}}\right)-\frac{1}{2}\left[\frac{(-1)^{i}\|\boldsymbol{\mu}\|^{2}}{\sigma^{2}} \bar{G}(\mathbf{w})-\frac{p}{n_{0}}+\frac{p}{n_{1}}\right] \stackrel{a . s}{\longrightarrow} 0,
$$




$$
D\left(\boldsymbol{\mu}_{i}, \overline{\mathbf{x}}_{0}, \overline{\mathbf{x}}_{1}, \hat{\mathbf{C}}, \boldsymbol{\Sigma}\right)-\left[\frac{\|\boldsymbol{\mu}\|^{2}}{\sigma^{2}} \bar{D}(\mathbf{w})+\frac{p}{n_{0}}+\frac{p}{n_{1}}\right] \stackrel{a . s}{\longrightarrow} 0,
$$

where

$$
\begin{aligned}
& \bar{G}(\mathbf{w})=1+\sum_{j=1}^{r} a_{j} b_{j} w_{j} \\
& \bar{D}(\mathbf{w})=1+\sum_{j=1}^{r}\left[\lambda_{j} b_{j}+2 a_{j} b_{j}\left(\lambda_{j}+1\right) w_{j}\right] \\
& +\sum_{j=1}^{r}\left[a_{j} b_{j}\left(1+\lambda_{j} a_{j}\right) w_{j}^{2}\right]
\end{aligned}
$$

with

$\boldsymbol{\mu}=\boldsymbol{\mu}_{0}-\boldsymbol{\mu}_{1}, \quad a_{j}=\frac{1-c / \lambda_{j}^{2}}{1+c / \lambda_{j}}, \quad b_{j}=\frac{\boldsymbol{\mu}^{T} \mathbf{u}_{j} \mathbf{u}_{j}^{T} \boldsymbol{\mu}}{\|\boldsymbol{\mu}\|^{2}}, \quad j=1, \cdots, r$

Proof: The proof is omitted for space limitations.

Using these deterministic equivalents and after some manipulations, a deterministic equivalent of the global classification error can be obtained as:

$$
\mathbb{E}\left[\epsilon^{\mathrm{Imp}-\mathrm{LDA}}\right]-\bar{\epsilon}^{\mathrm{Imp}-\mathrm{LDA}} \rightarrow 0 .
$$

where

$$
\bar{\epsilon}^{\operatorname{Imp}-\mathrm{LDA}}=\pi_{0} \boldsymbol{\Phi}\left(\frac{-\alpha(\bar{G}(\mathbf{w})-\eta)}{\sqrt{\bar{D}(\mathbf{w})+\omega}}\right)+\pi_{1} \boldsymbol{\Phi}\left(\frac{-\alpha(\bar{G}(\mathbf{w})+\eta)}{\sqrt{\bar{D}(\mathbf{w})+\omega}}\right)
$$

with $\alpha=\frac{\|\boldsymbol{\mu}\|}{2 \sigma}, \eta=\frac{\sigma^{2}}{\|\boldsymbol{\mu}\|^{2}}\left[c / \pi_{0}-c / \pi_{1}+2 \log \frac{\pi_{1}}{\pi_{0}}\right]$ and $\omega=$ $\frac{\sigma^{2}}{\|\boldsymbol{\mu}\|^{2}}\left[p / n_{0}+p / n_{1}\right]$. Now, with the asymptotic equivalent of the total classification error on hand, we can determine the optimal parameters $w_{j}^{\star}$.

Theorem 2. Under the settings of Assumption 1, the optimal parameters $\left\{w_{j}^{\star}\right\}$ that minimize $\bar{\epsilon}^{\mathrm{Imp}-\mathrm{LDA}}$ are given by:

$$
w_{j}^{\star}=\frac{u^{\star}}{\beta} \frac{\gamma_{j}}{\alpha_{j}}-\beta_{j}, \quad j=1, \cdots, r
$$

where

$\alpha_{j}=\lambda_{j} a_{j}^{2} b_{j}+a_{j} b_{j}, \quad \beta_{j}=\frac{\lambda_{j}+1}{\lambda_{j} a_{j}+1}, \quad \gamma_{j}=a_{j} b_{j}, \quad j=1, \ldots, r$,

$\beta=\sqrt{\sum_{j=1}^{r} \gamma_{j}^{2} / \alpha_{j}}$, and $u^{\star}$ is the unique positive solution of the following equation: $h(u)=0$, where

$h(u)=\pi_{0}\left(\beta b-d_{0} u\right) e^{\frac{-\alpha^{2}}{2} \frac{\left(\beta u+d_{0}\right)^{2}}{u^{2}+b}}+\pi_{1}\left(\beta b-d_{1} u\right) e^{\frac{-\alpha^{2}}{2} \frac{\left(\beta u+d_{1}\right)^{2}}{u^{2}+b}}$,

with

$$
\begin{aligned}
b & =1+\omega+\sum_{j=1}^{r}\left[\lambda_{j} b_{j}-\frac{\left(\lambda_{j} a_{j} b_{j}+a_{j} b_{j}\right)^{2}}{\lambda_{j} a_{j}^{2} b_{j}+a_{j} b_{j}}\right], \\
d_{0} & =1-\eta-\sum_{j=1}^{r} \frac{\lambda_{j} a_{j} b_{j}+a_{j} b_{j}}{\lambda_{j} a_{j}+1}, \\
d_{1} & =1+\eta-\sum_{j=1}^{r} \frac{\lambda_{j} a_{j} b_{j}+a_{j} b_{j}}{\lambda_{j} a_{j}+1} .
\end{aligned}
$$

\section{Proof: See Appendix A.}

Remark 3. - The determination of the design parameters $w_{j}$ involve finding the unique positive zero $u^{*}$ of function $h(u)$ defined in (11). This can be numerically obtained using, for instance, a bisection algorithm with a few number of iterations since tight upper and lower bounds of $u^{*}$ can be easily obtained.

- In the case of equiprobable classes, $u^{\star}$ can be computed in closed form expression as $u^{\star}=\frac{\beta b}{d}$, where $d=1-$ $\sum_{j=1}^{r} \frac{\lambda_{j} a_{j} b_{j}+a_{j} b_{j}}{\lambda_{j} a_{j}+1}$.

The optimal design parameters in Theorem 2 could not be directly used in practice, since they depend on the unobservable quantities $\lambda_{j}$ and $b_{j}$. To solve this issue, consistent estimators for these quantities need to be retrieved. This is the objective of the following result:

Proposition 4. Under the settings of Assumption $1, \lambda_{j}>\sigma^{2}(1+$ $\sqrt{c})^{2}, j=1, \cdots, r$ with probability one and we have

$$
\left|\lambda_{j}-\hat{\lambda}_{j}\right| \stackrel{a . s}{\longrightarrow} 0, \quad \text { and } \quad\left|b_{j}-\hat{b}_{j}\right| \stackrel{a . s}{\longrightarrow} 0,
$$

where

$$
\begin{aligned}
& \hat{\lambda}_{j}=\frac{s_{j} / \sigma^{2}+1-c+\sqrt{\left(s_{j} / \sigma^{2}+1-c\right)^{2}-4 s_{j} / \sigma^{2}}}{2} \\
& \hat{b}_{j}=\frac{1+c / \hat{\lambda}_{j}}{1-c / \hat{\lambda}_{j}^{2}} \frac{\hat{\boldsymbol{\mu}}^{T} \mathbf{v}_{j} \mathbf{v}_{j}^{T} \hat{\boldsymbol{\mu}}}{\|\hat{\boldsymbol{\mu}}\|^{2}}, \quad j=1, \cdots, r .
\end{aligned}
$$

with $\hat{\boldsymbol{\mu}}=\overline{\mathbf{x}}_{0}-\overline{\mathbf{x}}_{1}$ and $s_{j}$ is the $j$-th largest eigenvalue of the pooled covariance matrix.

Proof: The proof is a direct application of results from [24], [25] and it is thus omitted.

Replacing $\lambda_{j}$ and $b_{j}$ by their estimates yields a consistent estimator $\hat{w}_{j}^{\star}$ of $w_{j}^{\star}$.

\section{NUMERICAL SimUlations}

In this section, we study the performance of the proposed improved LDA classifier. We compare its performance with RLDA classifier for both synthetic and real data.

\section{A. Synthetic data}

In the synthetic data simulations, we use the following protocol for Monte Carlo estimation of the true classification error:

- Step 1: Set $\sigma^{2}=1$ and choose $r=3$ orthogonal symmetry breaking directions as follows : $\mathbf{u}_{1}=[1,0, \cdots, 0]^{T}$, $\mathbf{u}_{2}=[0,1,0, \cdots, 0]^{T}, \mathbf{u}_{3}=[0,0,1,0 \cdots, 0]^{T}$ and their corresponding weights $\lambda_{1}=4, \lambda_{2}=3, \lambda_{3}=2$. Set $\boldsymbol{\mu}_{0}=\frac{1}{\sqrt{p}}[a, a, \cdots, a]^{T}$ and $\boldsymbol{\mu}_{1}=-\boldsymbol{\mu}_{0}$ where $a$ is a finite constant. We choose $a=1$ and $a=2.5$.

- Step 2: Generate $n_{i}$ training samples for class $i$.

- Step 3: Using the training data set, design the improved LDA classifier as explained in section III and determine the optimal parameter $\gamma^{*}$ of R-LDA using a grid search over $\gamma \in$ $\left\{10^{i / 10}, i=-10: 1: 10\right\}$.

- Step 4: Compute the true error of both classifiers in each class using (5).

- Step 5: Repeat Step 2-4, 500 times and determine the average classification true error of both classifiers.

In Fig. 1, we plot the classification error vs. training sample size $n$ when $p=150$ and $\pi_{0}=\pi_{1}=1 / 2$ for the proposed improved LDA and the classical RLDA using synthetic data. It is observed that the improved LDA outperforms the classical R-LDA with a significant gain in classification performance. 


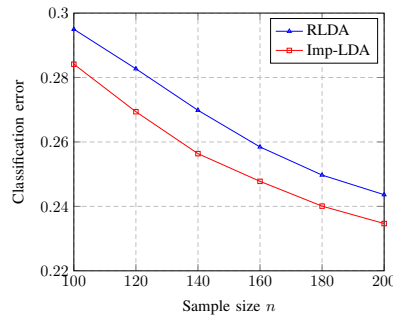

(a) $a=1$

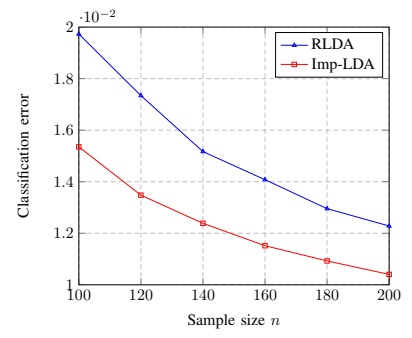

(b) $a=2.5$
Fig. 1: Classification error vs. sample size $n$ for $p=150$ and $\pi_{0}=$ $\pi_{1}=1 / 2$. Comparison between Improved LDA and RLDA with synthetic data.

\section{B. Real data}

For real data simulation, we use the "USPS" dataset which is one of the standard datasets for handwritten digit recognition. The dataset is publicly available at http://www.csie.ntu.edu.tw/ cjlin/libsvmtools/ datasets. For this dataset, the number of features (observation size) is $p=256$ and the total number of samples for all digits including test samples is 9298 . We use the following protocol for the real dataset:

- Step 1: Let $q_{0}$ be the ratio between the total number of samples in class $\mathcal{C}_{0}$ to the total number of samples available in the full dataset. Denote by $n_{\text {Full }}$ the total number of samples in the full dataset. Choose $n<n_{\text {Full }}$ the number of training samples; set $n_{0}=\left\lfloor q_{0} n\right\rfloor$, where $\lfloor$.$\rfloor is the floor function and n_{1}=n-n_{0}$. Take $n_{i}$ training samples belonging to class $\mathcal{C}_{i}$ randomly from the full dataset. The remaining samples are used as a test dataset in order to estimate the classification error.

- Step 2: Using the training dataset, design the improved LDA classifier as explained in section III and determine the optimal parameter $\gamma^{*}$ of R-LDA using a grid search over $\gamma \in$ $\left\{10^{i / 10}, i=-10: 1: 10\right\}$

- Step 3: Using the test dataset, estimate the true classification error for both classifiers.

- Step 4: Repeat steps 1-4, 500 times and determine the average classification error for both classifiers.

In Fig. 2, the classification error vs. training sample size is plotted for the RLDA and our proposed classifier. Three pairs of the most confusing digits are chosen for simulation; $(3,8),(4,9)$ and $(8,9)$. As seen, the proposed classifier presents a higher gain over the R-LDA classifier. For instance, more that $10 \%$ gain in terms of classification error rate is obtained for digits $(4,9)$ when $n=1000$.

\section{CONCLUSION}

In this paper, we proposed a novel LDA classifier that is optimally conceived for high dimensional settings in which the number of observations scales with their dimensions. The proposed technique is based on an improved estimator of the inverse covariance matrix, the parameters of which are carefully chosen to minimize the asymptotic misclassification error rate. Interestingly, the proposed estimator is not only easy to implement but is also shown to outperform the classical LDA and R-LDA classifiers over both synthetic and real data sets. This work opens up several research directions to be pursued in the future. For example, it would be interesting to apply the same approach to the quadratic discriminant analysis (QDA) classifier. In addition, more involved spiked models may be considered in which the population covariance matrix is low-rank perturbation of a diagonal matrix.

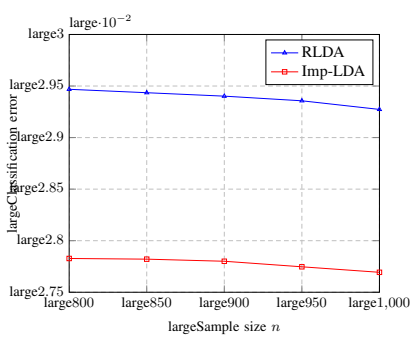

(a) Digits 3 and 8

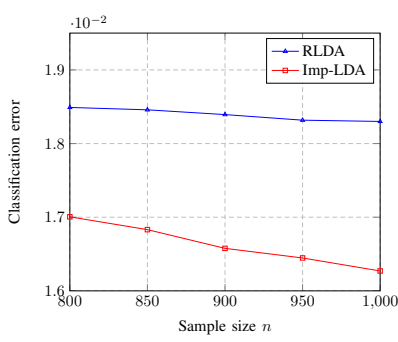

(b) Digits 4 and 9

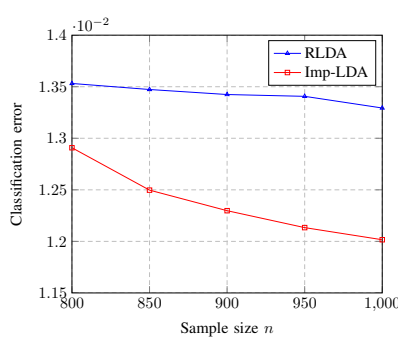

(c) Digits 8 and 9

Fig. 2: Classification error vs. sample size $n$. Comparison between Improved LDA and RLDA using USPS dataset.

\section{APPENDIX A}

PROOF OF THEOREM 2

The objective function is given by:

$$
f(\mathbf{w})=\pi_{0} \mathbf{\Phi}\left(-\alpha f_{0}(\mathbf{w})\right)+\pi_{1} \mathbf{\Phi}\left(-\alpha f_{1}(\mathbf{w})\right),
$$

where

$$
f_{0}(\mathbf{w})=\frac{\bar{G}(\mathbf{w})-\eta}{\sqrt{\bar{D}(\mathbf{w})+\omega}} \text { and } f_{1}(\mathbf{w})=\frac{\bar{G}(\mathbf{w})+\eta}{\sqrt{\bar{D}(\mathbf{w})+\omega}}
$$

We have,

$$
f_{0}(\mathbf{w})=\frac{1-\eta+\sum_{j=1}^{r} a_{j} b_{j} w_{j}}{\sqrt{1+\omega+\sum_{j=1}^{r} b_{j}\left[\lambda_{j}+a_{j}\left(2\left(\lambda_{j}+1\right) w_{j}+\left(1+\lambda_{j} a_{j}\right) w_{j}^{2}\right)\right]}}
$$

The numerator of $f_{0}(\mathbf{w})$ can be rewritten as:

$$
\sum_{j=1}^{r}\left[a_{j} b_{j}\left(w_{j}+\frac{\lambda_{j}+1}{\lambda_{j} a_{j}+1}\right)-\frac{\lambda_{j} a_{j} b_{j}+a_{j} b_{j}}{\lambda_{j} a_{j}+1}\right]+1-\eta
$$

And the square of the denominator of $f_{0}(\mathbf{w})$ can be expressed as:

$$
\begin{aligned}
1+\omega+\sum_{j=1}^{r} & {\left[\left(\lambda_{j} a_{j}^{2} b_{j}+a_{j} b_{j}\right)\left(w_{j}+\frac{\lambda_{j}+1}{\lambda_{j} a_{j}+1}\right)^{2}\right.} \\
& \left.+\lambda_{j} b_{j}-\frac{\left(\lambda_{j} a_{j} b_{j}+a_{j} b_{j}\right)^{2}}{\lambda_{j} a_{j}^{2} b_{j}+a_{j} b_{j}}\right]
\end{aligned}
$$

Thus, $f_{0}(\mathbf{w})$ can be rewritten as:

$$
f_{0}(\mathbf{w})=\frac{\sum_{j=1}^{r} \gamma_{j}\left(w_{j}+\beta_{j}\right)+d_{0}}{\sqrt{\sum_{j=1}^{r} \alpha_{j}\left(w_{j}+\beta_{j}\right)^{2}+b}}
$$

where

$$
\alpha_{j}=\lambda_{j} a_{j}^{2} b_{j}+a_{j} b_{j}, \quad \beta_{j}=\frac{\lambda_{j}+1}{\lambda_{j} a_{j}+1}, \quad \gamma_{j}=a_{j} b_{j}, \quad j=1, \ldots, r
$$


and

$$
\begin{aligned}
b & =1+\omega+\sum_{j=1}^{r}\left[\lambda_{j} b_{j}-\frac{\left(\lambda_{j} a_{j} b_{j}+a_{j} b_{j}\right)^{2}}{\lambda_{j} a_{j}^{2} b_{j}+a_{j} b_{j}}\right], \\
d_{0} & =1-\eta-\sum_{j=1}^{r} \frac{\lambda_{j} a_{j} b_{j}+a_{j} b_{j}}{\lambda_{j} a_{j}+1} .
\end{aligned}
$$

Then, we have

$$
f_{0}(\mathbf{w})=\frac{\mathbf{c}^{T} \tilde{\mathbf{w}}+d_{0}}{\sqrt{\tilde{\mathbf{w}}^{T} \mathbf{D} \tilde{\mathbf{w}}+b}}
$$

where the elements of $\tilde{\mathbf{w}}$ are $\tilde{w}_{j}=w_{j}+\beta_{j}, \mathbf{c}=\left[\gamma_{1}, \cdots, \gamma_{r}\right]^{T}$ and $\mathbf{D}=\operatorname{diag}\left(\alpha_{1}, \cdots, \alpha_{r}\right)$. Similarly, it can be shown that:

$$
f_{1}(\mathbf{w})=\frac{\mathbf{c}^{T} \tilde{\mathbf{w}}+d_{1}}{\sqrt{\tilde{\mathbf{w}}^{T} \mathbf{D} \tilde{\mathbf{w}}+b}}
$$

where

$$
d_{1}=1+\eta-\sum_{j=1}^{r} \frac{\lambda_{j} a_{j} b_{j}+a_{j} b_{j}}{\lambda_{j} a_{j}+1}
$$

Our objective function can be rewritten as:

$$
f(\tilde{\mathbf{w}})=\pi_{0} \boldsymbol{\Phi}\left(\frac{-\alpha\left(\mathbf{c}^{T} \tilde{\mathbf{w}}+d_{0}\right)}{\sqrt{\tilde{\mathbf{w}}^{T} \mathbf{D} \tilde{\mathbf{w}}+b}}\right)+\pi_{1} \boldsymbol{\Phi}\left(\frac{-\alpha\left(\mathbf{c}^{T} \tilde{\mathbf{w}}+d_{1}\right)}{\sqrt{\tilde{\mathbf{w}}^{T} \mathbf{D} \tilde{\mathbf{w}}+b}}\right) .
$$

Letting $u=\left\|\mathbf{D}^{\frac{1}{2}} \tilde{\mathbf{w}}\right\|$ and $\overline{\mathbf{w}}=\frac{\mathbf{D}^{\frac{1}{2}} \tilde{\mathbf{w}}}{\left\|\mathbf{D}^{\frac{1}{2}} \tilde{\mathbf{w}}\right\|}$, we have

$$
\min _{\tilde{\mathbf{w}}} f(\tilde{\mathbf{w}})=\min _{\substack{(\overline{\mathbf{w}}, u) \\\|\overline{\mathbf{w}}\|=1, u>0}} g(\overline{\mathbf{w}}, u)=\min _{\substack{u \\ u>0\|\overline{\mathbf{w}}\|=1}} \min _{\substack{\overline{\mathbf{w}}\\}} g(\overline{\mathbf{w}}, u),
$$

where

$$
\begin{aligned}
g(\overline{\mathbf{w}}, u) & =\pi_{0} \boldsymbol{\Phi}\left(\frac{-\alpha\left(\mathbf{c}^{T} \mathbf{D}^{-\frac{1}{2}} \overline{\mathbf{w}} u+d_{0}\right)}{\sqrt{u^{2}+b}}\right) \\
& +\pi_{1} \boldsymbol{\Phi}\left(\frac{-\alpha\left(\mathbf{c}^{T} \mathbf{D}^{-\frac{1}{2}} \overline{\mathbf{w}} u+d_{1}\right)}{\sqrt{u^{2}+b}}\right) .
\end{aligned}
$$

Since $u>0$ and $\boldsymbol{\Phi}()$ is an increasing function, $\overline{\mathbf{w}}^{\star}$ that minimizes $g(\overline{\mathbf{w}}, u)$ subject to $\|\overline{\mathbf{w}}\|=1$ is the minimizer of $-\mathbf{c}^{T} \mathbf{D}^{-\frac{1}{2}} \overline{\mathbf{w}}$ subject to $\|\overline{\mathbf{w}}\|=1$. Thus, $\overline{\mathbf{w}}^{\star}=\frac{1}{\sqrt{\mathbf{c}^{T} \mathbf{D}^{-1} \mathbf{c}}} \mathbf{D}^{-\frac{1}{2}} \mathbf{c}$. Replacing $\overline{\mathbf{w}}^{\star}$ in $g(\overline{\mathbf{w}}, u)$ yields,

$$
\tilde{g}(u)=\pi_{0} \boldsymbol{\Phi}\left(\frac{-\alpha\left(\beta u+d_{0}\right)}{\sqrt{u^{2}+b}}\right)+\pi_{1} \boldsymbol{\Phi}\left(\frac{-\alpha\left(\beta u+d_{1}\right)}{\sqrt{u^{2}+b}}\right),
$$

where $\beta=\sqrt{\mathbf{c}^{T} \mathbf{D}^{-1} \mathbf{c}}=\sqrt{\sum_{j=1}^{r} \gamma_{j}^{2} / \alpha_{j}}$. It remains now to determine the minimizer of $\tilde{g}(u)$. To this end, we investigate the derivative of $\tilde{g}(u)$ :

$$
\tilde{g}^{\prime}(u)=\frac{-\alpha}{\sqrt{2 \pi}\left(u^{2}+b\right)^{\frac{3}{2}}} h(u),
$$

where

$h(u)=\pi_{0}\left(\beta b-d_{0} u\right) e^{\frac{-\alpha^{2}}{2} \frac{\left(\beta u+d_{0}\right)^{2}}{u^{2}+b}}+\pi_{1}\left(\beta b-d_{1} u\right) e^{\frac{-\alpha^{2}}{2} \frac{\left(\beta u+d_{1}\right)^{2}}{u^{2}+b}}$.

It can be shown using complex analysis that $h(u)$ has a unique positive zero. The details are omitted for space limitations. Let $u^{\star}>0$ be the unique positive solution of the following equation:

$$
h(u)=0 .
$$

Then, we have

$$
\tilde{g}^{\prime}\left(u^{\star}\right)=0, \quad \tilde{g}^{\prime}(u)<0 \text { for } u<u^{\star}, \quad \tilde{g}^{\prime}(u)>0 \text { for } u>u^{\star} .
$$

This implies that $\tilde{g}(u)$ has a unique minimizer which is $u^{\star}$. Finally, we have $\tilde{\mathbf{w}}^{\star}=u^{\star} \mathbf{D}^{-\frac{1}{2}} \overline{\mathbf{w}}^{\star}=\frac{u^{\star}}{\sqrt{\mathbf{c}^{T} \mathbf{D}^{-1} \mathbf{c}}} \mathbf{D}^{-1} \mathbf{c}$.

\section{REFERENCES}

[1] R. A. Fisher, "The use of multiple measurements in taxonomic problems," Ann. Eugen., vol. 7, no. 2, pp. 179-188, 1936.

[2] K. R. Varshney, "Generalization error of linear discriminant analysis in spatially-correlated sensor networks," IEEE Transactions on Signal Processing, vol. 60, no. 6, pp. 3295-3301, Jun. 2012.

[3] S. van Vuuren and H. Hermansky, "Data-driven design of RASTA-like filters," in Proc. Eurospeech, 1997, pp. 409-412.

[4] D. L. Swets and J. J. Weng, "Using discriminant eigenfeatures for image retrieval," IEEE Transactions on Pattern Analysis and Machine Intelligence, vol. 18, no. 8, pp. 831-836, Aug. 1996.

[5] S. Kim, E. R. Dougherty, I. Shmulevich, K. R. Hess, S. R. Hamilton, J. M. Trent, G. N. Fuller, and W. Zhang, "Identification of combination gene sets for glioma classification," Mol. Cancer Ther, vol. 1, no. 13, pp. 1229-1236, 2002.

[6] T. Hastie, R. Tibshirani, and J. Friedman, The Elements of Statistical Learning, Springer, 2001.

[7] A. Zollanvari and E. R. Dougherty, "Generalized consistent error estimator of linear discriminant analysis," IEEE Transactions on Signal Processing, vol. 63, no. 11, pp. 2804-2814, Jun. 2015.

[8] Daniyar Bakirov, Alex Pappachen James, and Amin Zollanvari, "An efficient method to estimate the optimum regularization parameter in RLDA," Bioinformatics, vol. 32, no. 22, pp. 3461-3468, 2016.

[9] Khalil Elkhalil, Abla Kammoun, Romain Couillet, Tareq Y. AlNaffouri, and Mohamed-Slim Alouini, "A Large Dimensional Study of Regularized Discriminant Analysis Classifiers," https://arxiv.org/abs/1711.00382, 2017.

[10] D. C. Hoyle and M. Rattray, "Limiting form of the sample covariance eigenspectrum in PCA and kernel PCA," Proceedings of Neural Information Processing Systems, 2003.

[11] P. Reimann, C. Van den Broeck, and G.J. Bex, "A gaussian scenario for unsupervised learning," J. Phys. A:Math. Gen., 1996.

[12] C. M. Bishop, Pattern Recognition and Machine Learning, Springer, 2006.

[13] K. Elkhalil, A. Kammoun, R. Couillet, T. Y. Al-Naffouri, and M. S. Alouini, "Asymptotic performance of regularized quadratic discriminant analysis based classifiers," in IEEE 27th International Workshop on Machine Learning for Signal Processing (MLSP), Sept. 2017, pp. 1-6.

[14] E. Dobriban and S. Wager, "High-dimensional asymptotics of prediction: ridge regression and classification," The Annals of Statistics, vol. 46, no. 1, pp. 247-279, 2018.

[15] S. Kritchman and B. Nadler, "Determining the number of components in a factor model from limited noisy data," Chemometrics and Intelligent Laboratory Systems, vol. 94, no. 1, pp. 19 - 32, 2008.

[16] I. M. Johnstone and A. Y. Lu, "On consistency and sparsity for principal components analysis in high dimensions," J. Amer. Stat. Assoc., vol. 104, no. 486, pp. 682-693, 2009.

[17] M. O. Ulfarsson and V. Solo, "Dimension estimation in noisy pca with sure and random matrix theory," IEEE Transactions on Signal Processing, vol. 56, no. 12, pp. 5804-5816, Dec. 2008.

[18] D. Passemier, Z. Li, and J. Yao, "On estimation of the noise variance in high dimensional probabilistic principal component analysis," Journal of the Royal Statistical Society: Series B (Statistical Methodology), vol. 79, no. 1, pp. 51-67, 2017.

[19] M. J. Daniels and R. E. Kass, "Shrinkage estimators for covariance matrices," Biometrics, vol. 57, no. 4, pp. 1173-1184, Dec. 2001.

[20] O. Ledoit and M. Wolf, "A well-conditioned estimator for largedimensional covariance matrices," Journal of Multivariate Analysis, vol. 88 , no. 2 , pp. $365-411,2004$.

[21] N. El Karoui, "Spectrum estimation for large dimensional covariance matrices using random matrix theory," Ann. Statist., vol. 36, no. 6, pp. 2757-2790, 2018.

[22] Y. Chen, A. Wiesel, Y. C. Eldar, and A. O. Hero, "Shrinkage algorithms for mmse covariance estimation," IEEE Transactions on Signal Processing, vol. 58, no. 10, pp. 5016-5029, Oct. 2010.

[23] O. Ledoit and M. Wolf, "Nonlinear shrinkage of the covariance matrix for portfolio selection: Markowitz meets goldilocks," The Review of Financial Studies, vol. 30, no. 12, pp. 4349-4388, 2017.

[24] T. Couillet and M. Debbah, Random Matrix Methods for Wireless Communications, U.K., Cambridge: Cambridge Univ. Press, 2011.

[25] J. Baik, G. Ben Arous, and S. Péché, "Phase transition of the largest eigenvalue for nonnull complex sample covariance matrices," Ann. Probab., vol. 33, no. 5, pp. 1643-1697, Sept. 2005. 